\title{
Seeing is believing? A mixed methods study exploring the quality and perceived trustworthiness of online information about chronic conditions aimed at children and young people
}

\section{[original paper]}

Amy C McPherson*, PhD, CPsychol, AFBPsS

Scientist, Bloorview Research Institute, Holland Bloorview Kids Rehabilitation Hospital

Assistant Professor, Dalla Lana School of Public Health, University of Toronto.

* Corresponding author:

150 Kilgour Road, Toronto, ON, Canada. M4G 1R8

E-mail: amcpherson@hollandbloorview.ca

Tel: (001) 416-425-6220 ext. 6378

Fax: (001) 416-422-7037

Miriam L. Gofine, BSc.

Bloorview Research Institute, Holland Bloorview Kids Rehabilitation Hospital

Jennifer Stinson, RN, PhD, CPNP

Child Health Sciences, The Hospital for Sick Children

Lawrence S. Bloomberg Faculty of Nursing, University of Toronto 


\begin{abstract}
Background

The numbers of children and young people with chronic conditions are increasing. Whilst their information needs may vary, providing healthcare information can have considerable benefits, including better emotional health, less distress during treatments, as well as greater satisfaction with medical care. The Internet is increasingly being used to communicate healthrelated information to children about a range of chronic conditions. However, the quality of such websites is under-explored.
\end{abstract}

\title{
Objectives
}

To evaluate the reliability and quality of online information for children about chronic conditions using a standardised evaluation tool, and to explore children and young people’s perceptions of quality and trustworthiness regarding online health information.

\section{Method}

The study consisted of two phases. In Phase 1, websites about common paediatric chronic conditions aimed at children and containing treatment or management options were identified and the quality assessment tool DISCERN completed. Test-retest and inter-rater reliability were calculated. In Phase 2, two focus groups with laptop computers were conducted with children and young people with a chronic condition to explore their perceptions of trustworthiness of online health information. 


\section{Results}

In Phase 1, 165 websites were identified and 100 met the criteria and were assessed. The mean DISCERN score of all sites was 48.16 out of 75 (SD=7.97, range 28-71, min 15 - max 75). Quality scores varied widely across the sample. The internal consistency and inter-rater reliability scores were both lower than previously reported in studies using the DISCERN to assess information for adults. Two focus groups with a total of six participants aged 11-23 years revealed a relative lack of concern about the quality and trustworthiness of online health information. Older participants reported judging the source and authorship of websites, but other participants did not question the source of the information they found online. Although personal websites were perceived to be less reliable than those from well-known medical institutions, they were still valued by many of the participants.

\section{Discussion}

There were relatively few websites about paediatric chronic conditions aimed at children, with variable quality and reliability. However, the DISCERN's use with paediatric websites across a broad spectrum of developmental stages is limited. Children and young people demonstrated a need for more guidance around assessing trustworthiness of online information. A more appropriate quality assessment tool is warranted, which could usefully be employed by healthcare professionals, children and parents. 
The numbers of children and young people with chronic conditions are increasing (Sawyer, Drew, Yeo, \& Britto, 2007). Providing relevant healthcare information to these children has considerable benefits, including better emotional health, less distress during treatment, and greater satisfaction with medical care (Veldtman et al., 2000; Rushforth, 1999; O'Connor, 2009). A better understanding of their condition also facilitates a greater sense of autonomy over health, with positive implications for subsequent self-management (Tieffenberg, Wood, Alonso, Tossutti, \& Vicente, 2000). Receiving information about chronic conditions can also result in decreased emergency room visits, days of hospitalization, healthcare costs, morbidity, and need for additional support (Dunn \& Austin, 2004; Pantell \& Lewis, 1993). In addition, siblings and peers may benefit from learning about chronic conditions and their management (Barlow \& Ellard, 2004).

However, varying literacy and cognitive abilities may limit the use of traditional written information. Healthcare providers have therefore attempted to harness the Internet to provide health-related information to children both with and without chronic conditions, recognizing that children are increasingly using it as a portal to information about health and wellness (Gray, Klein, Noyce, Sesselberg, \& Cantrill, 2005a; Franck, Noble, \& McEvoy, 2008). For example, English-speaking teens in the UK and US reported the Internet to be their primary health information source, regardless of socio-economic status (Gray et al, 2005a). The Internet is often particularly used for sensitive or stigmatizing issues, such as sexual health (Buhi et al, 2010).

An additional benefit is that the Internet can effectively convey complex concepts in a simplified manner, for example, by using voiceovers and animation (McPherson, Glazebrook, \& Smyth, 2005; Wong \& Tam, 2010). It has been hypothesized that this allows children to extend beyond their existing knowledge to a more sophisticated level of understanding, termed 
"scaffolding” (Wood, 1998). Thus, a computer program or website that provides simplified instructions and simulations of complex physiological processes, can potentially help children understand more complex aspects of health. Furthermore, both personalizing programs according to the user's preferences and presenting information in the context of games and other interactive activities - termed 'edutainment' (Noriaki, Sachiko, Taisuke, Oishi, \& Tsuguya, 2005)- can encourage longer engagement with the material (Brown, Lieberman, Gemeny et al, 1997; Montgomery \& Chester, 2009). Using a variety of transmission modes (e.g. illustrations, animation and interactive games) to provide online health information to children is therefore likely to lead to a higher quality learning experience that would be unavailable via a website aimed at adults. Crucially, skills taught in a 'virtual' environment have been shown to generalize to situations beyond the computer (Standen, 1998; McPherson, Glazebrook, Forster, James, \& Smyth, 2006).

The Internet can also promote the inclusion of people with disabilities who would otherwise be marginalized by traditional educational approaches (Blair, 2006; Stewart, Barnfather, Magill-Evans, Ray, \& Letourneau, 2011). Despite accessibility concerns, recent studies report high levels of Internet access for children both with and without chronic conditions (Lathouwers, de Moor, \& Didden, 2009);. In the US, Canada and the UK, young people’s access to the Internet is almost universal, with figures ranging from 93\% to 98\% (The Pew Foundation, 2010; Erin Research Inc., 2005; Livingstone \& Bober, 2005). Furthermore, some youth with chronic conditions may be even more likely than their peers to seek healthcare information online (Suris, Akre, Berchtold, Belanger, \& Michaud, 2010).

Reliable, high-quality online healthcare information is therefore required. However, evaluations of adult online health information indicate that the quality varies and is typically 
poor (Al-Bahrani \& Plusa, 2004; Kaicker, Borg Debono, Dang, Buckley, \& Thabane, 2010). The quality of websites about paediatric conditions specifically aimed at children and young people (rather than their parents) has been less extensively studied, although there are indications that their quality also varies (Hargrave, Hargrave, \& Bouffet, 2010; Bergman, Konijeti, \& Lerman, 2007). This is especially concerning given that many parents do not advise their children about assessing the reliability of online information (Lathouwers et al., 2009; Livingstone et al., 2005) and children may have difficulty establishing the trustworthiness of websites (Gray, Klein, Noyce, Sesselberg, \& Cantrill, 2005b; Hansen, Derry, Resnick, \& Richardson, 2003; Livingstone \& Bober, 2005). Although healthcare professionals may be able to evaluate the quality and reliability of healthcare information intuitively given their training, this can be a time-consuming task if evaluating multiple websites.

There is also a paucity of reliable and valid instruments to assess the quality of online paediatric information (Hanif, Read, Goodacre, Chaudhry, \& Gibbs, 2009). To evaluate information aimed at adults, some researchers have developed their own guidelines, either independently (e.g. Ilic, Risbridger, \& Green, 2004; Kunst, 2002;Silberg, Lundberg, \& Musacchio RA, 1997) or by adapting criteria developed by the American Medical Association or Health on the Net Foundation (e.g. Stieb, Wang, \& Haver, 2002; Anselmo, Lash, Stieb, \& Haver, 2004). Generally accepted indicators of quality include: (i) clear statement of the purpose and target audience for the web site; (ii) information about authorship, disclosure, and attribution; (iii) citation of best evidence; (iv) acknowledgement of risks, other treatments and the effect of no treatment; (v) references to other sources; and (vi) clear disclosure of sponsorship, affiliations, or conflicts of interest (Agency for Health Care Policy and Research, 2009; Kim et al., 1999). However, no published, validated scales have been developed specifically for websites targeting 
children and youth. The DISCERN (http://www.dis- cern.org.uk), was developed jointly by healthcare consumers and professionals to assess the reliability and quality of written information about treatment choices for adults (Barnes et al., 2003; Charnock \& Shepperd, 2004) and has also been used to assess online healthcare information (Charnock, Shepperd, Needham, \& Gann, 1999; Stinson et al., 2009; Ilic et al., 2004; Ademiluyi, Rees, \& Sheard, 2003). However, few studies have reported using the DISCERN to evaluate online information aimed at children (Stinson et al., 2011).

This study has two phases: Phase 1 uses the DISCERN tool to evaluate the reliability and quality of online healthcare information about chronic paediatric conditions specifically aimed at children and young people. Phase 2 is an initial exploration of the perceptions of children and young people about indicators of quality and trustworthiness of online healthcare information.

\section{Methods}

\section{Phase 1: Use of DISCERN}

Search strategy: The search for websites was performed in May and June 2010 (updated July 2012) using the online search engines Google and Yahoo, snowball sampling (following links from one page to another), and by directly requesting online resources from relevant organisations. The most common paediatric chronic conditions in the general population (Torpy, Lynm, \& Glass, 2007; Yeo \& Sawyer, 2005) were used as search terms in the search engines, which included (but were not limited to) asthma, diabetes (type I and type II), epilepsy, autism, Asperger's syndrome, scoliosis, cerebral palsy, and spina bifida and hydrocephalus. These search terms were combined with 'kids', ‘children', "I have [condition name]", “support” and “information”. 
Inclusion criteria: Websites had to be aimed specifically at children or youth (as stated on the website), both those with a chronic condition or their peers (e.g. friends, siblings etc). Websites which did not explicitly state that they were aimed at children/young people were excluded. To complete the DISCERN, the website also had to include treatment or management options. Websites about rare conditions (as stated on the website) were excluded as they may have differed substantially from websites for more typical conditions. Sites that could not be navigated (e.g. broken hyperlinks, loading problems), provided insufficient information to reliably complete the DISCERN, provided social support and/or social networking only and personal websites were also excluded. So too were sites providing information about acute illnesses (e.g. influenza) or only in formats such as online pamphlets or magazines. Interactive games and animation were included if they contained relevant content.

Instrument information and assessment: The DISCERN tool (see Table 1 for a listing of questions) uses 15 key criteria: the first section (questions 1-8) evaluates the reliability/trustworthiness of the information and the second section (questions 9-15) considers the quality of the information. Each item is rated on a 5-point Likert scale. A summary score is generated by summing the scores for each item, ranging from 15 to 75, with high scores indicating good quality (Charnock et al, 1999; Charnock \& Shepperd, 2010). A final question rates the overall quality of the treatment information on a Likert scale of $1-5$, which has 1 as “Low (serious or extensive shortcomings”) and 5 as "High (minimal shortcomings)”. In a previous study, the overall quality of websites was categorized as excellent (scores of 63-75), good (51-62), fair (39-50), poor (27-38), and very poor (15-26) (Lau, Hargrave, Bartels, Esquembre \& Buffet, 2006). Stinson et al (2011) has previously set a minimum acceptance DISCERN score of $>50 \%(37.5 / 75)$. 


\section{INSERT TABLE 1 HERE.}

Procedure: One rater (MG) conducted the searches, applied the inclusion/exclusion criteria and rated all sites using the DISCERN. The same rater re-assessed $25 \%$ of the sites after two weeks. Another rater (AM) independently assessed 25\% of the sites to calculate inter-rater reliability. Both raters used the structured guidance provided on the DISCERN website when completing the assessments.

Statistical analyses: Sections 1 and 2 of the DISCERN were summed both separately and together, which created one overall score between 15 and 75. Question 16 (overall quality) was analyzed separately. SPSS Version 16.0 was used to analyze the data. The KolmogorovSmirnov test was used to determine the distribution of the data and then parametric or nonparametric statistics used as appropriate to describe the data and test for relationships. The internal consistency of DISCERN was established by calculating Cronbach's alpha ( $\alpha)$. Intra-and inter- rater reliabilities were calculated to ascertain agreement and consistency rating levels between raters. A p-value less than 0.05 was considered to be significant in all tests.

\section{Phase 2: Qualitative Focus Groups}

Sample: The sample was drawn from a large paediatric rehabilitation hospital in Toronto, Canada. Inclusion criteria were a) aged 10-23 years; b) have a long-term chronic condition or disability; c) be able to communicate in English either verbally or with a communication aid; d) have used a computer. Siblings were also eligible because we were interested in websites aimed at all children.

Recruitment: Therapeutic Recreation staff, who have contact with both inpatient and outpatient clients distributed information letters. Youth facilitators who support inpatients with 
computer access also identified appropriate clients. The researcher discussed the study with any child interested in participating and screened them for eligibility. Children/ young people who could describe the study information in their own words with reasonable accuracy, and demonstrated understanding of the purpose of the study, their role in it, the risks and benefits, confidentiality, and voluntariness of participation, were judged to demonstrate capacity and provided written consent. Where children did not demonstrate capacity to consent, parents provided signed consent and children were invited to sign an assent statement.

Procedure: Following ethical approval from the hospital's Research Ethics Board, two focus groups lasting approximately 90 minutes were held. Participants only attended one focus group. At the start of each focus group, researchers obtained consent/assent and gathered demographic and Internet usage information from participants. One researcher (AM) facilitated the semi-structured focus groups, posing focused, open-ended questions. The session started by exploring participants' use of the Internet in general, before moving onto their use of online health information. Positive and negative elements of online information were explored, and how they judged the quality and trustworthiness of online health information. Laptop computers were provided in the focus groups, along with 4-5 example health-related websites (identified during Phase 1) for participants’ viewing and feedback. These were selected to cover a range of conditions (e.g. spina bifida, peanut allergy and epilepsy) and were judged to contain some potentially useful discussion points. Participants did not formally evaluate or rate the selected websites; their purpose was to provide some concrete examples to prompt further discussion if the participants found abstract thinking challenging, depending upon their age and cognitive functioning. Participants were also free to use other websites to illustrate their views. The 
discussions were audio recorded and transcribed verbatim. A research assistant took detailed field notes and recorded contextual factors.

Analysis: Demographic data were analyzed using descriptive statistics to summarize the characteristics of the study participants. Thematic analysis from a relativist ontological approach (Swift \& Tischler, 2010) was undertaken on the focus groups transcripts using a systematic, iterative approach (Braun \& Clarke, 2006). The transcripts were reviewed independently by the first author and a Research Assistant using inductive coding to obtain an overall understanding of the data. Codes were then compared and contrasted, and organized into key themes and agreed through consensus. Themes were then merged, relabeled or split as necessary. Excerpts from the data were used to support themes and illustrate patterns in the perceptions of the participants, ensuring that no one person or idea was over-represented.

\section{Results}

\section{Phase 1: Use of DISCERN}

Website selection: One hundred and sixty-five websites were identified; 65 were excluded because they did not meet the inclusion criteria. See Table 2 for an index of website topics and frequencies.

\section{INSERT TABLE 2 ABOUT HERE.}

DISCERN scores: The scores of Section 1 (information reliability) were not normally distributed and had a median score of 31 out of 40 (range 16-39) with scores skewed towards poorer reliability. The scores of Section 2 (information quality) were normally distributed with a mean of 17.7 out of 35 (SD=6.0, range $=7-35)$ and skewed slightly to higher quality. The combined data from Sections 1 and 2 had a mean score of 48.16 (SD=7.97) with a range of 28-71 
(out of a possible score range of 15-75). The mean score for Question 16 was 3.20 out of 5 ( $\mathrm{SD}=1.10)$. Scores of Section 1 (reliability) across all websites were significantly higher than the mean scores of Section 2 (quality) $\left(U=487.00, N_{1}=100, N_{2}=100, p<0.01\right.$, two-tailed).

Reliability testing: Test-retest reliability was calculated for $25 \%$ of websites (re-rated two weeks apart) using a Pearson correlation $(\mathrm{r}=0.77, \mathrm{n}=25, \mathrm{p}<0.01)$. Internal consistency, measured by Cronbach’s alpha, was 0.64 . The calculations of inter-rater reliability revealed that absolute agreement was ICC $=0.33(95 \%$ CI $-0.01-0.62)$ and consistency between raters was ICC $=0.38$ (95\% CI 0.00-0.658).

\section{Phase 2: Qualitative Focus Groups}

Participants: Six children/youth (four females and two males) attended the focus groups; five were clients and one was a sibling. Two additional participants were not well enough to attend either workshop despite previously providing consent. It is unknown how many information sheets the Therapeutic Recreation staff distributed in total. The mean age of the participants was 16.7 years (11-23 years). All used computers at home (with Internet access) and rated themselves as ‘comfortable’ or 'very comfortable' using the Internet. The names below are pseudonyms (see Table 3 for participants’ demographics).

\section{INSERT TABLE 3 ABOUT HERE}

Usage of the Internet: Participants used the Internet mainly for communicating with friends and for school assignments, with younger participants also reporting playing games online. Participants almost universally used the search engine Google to search for information, followed by Yahoo. They did not state at what age they started accessing the Internet, but it 
appeared to be an integral part of their lifestyle, with all but one participant reporting five or more hours of Internet usage per week.

Participants described the central role that the Internet played in informing their school assignments. The online encyclopedia, Wikipedia, was identified as a frequently used resource, with Joel (14 years, sibling) saying “Wikipedia saves my life!” For educational purposes, they particularly valued the ease of finding detailed information online;

“If you wanna like learn about something, if you hear about something and you don't know what it is, you can just learn on a YouTube video” (Kristen, 18 years)

Not all of the participants reported using the Internet for health-related information, but some talked about how it could potentially augment information provided by a healthcare provider;

“...'cos I have a sleeping disorder...so I look up the medication...instead of reading the pamphlets they give” (Tabitha, 19)

This could be particularly important given the perceived brevity of some medical appointments;

"Since the...clinics are usually ten minutes and if you want more information you always nowadays, like you Google everything” (Catherine, 23 years)

It also provided a way to hear about other people’s experiences of health and illness; 
“...sometimes I watch YouTube videos about sick kids and stuff...like people’s stories... cancer stories, or like surgery stories or an illness that they had" (Kristen, 18 years)

A further use of the Internet was to facilitate conversations between peers about particular conditions. For example, Catherine (23 years) explained; “...my best friend has epilepsy, so we have done researching [sic] when we are together...”

Judging information quality: Participants selected information from websites using a number of criteria, which differed across ages. Younger participants prioritized relevance over reliability, concentrating upon the first few results of a web search; "Usually, the top 5, 6 are the best" (Connor, 11). They also reported paying particular attention to those results in which the search words appeared most often. Information described in a clear and straightforward manner was particularly sought, for example, "I like Wikipedia because I can click this button which says 'Simple English”' (Connor, 11). Older youth recognized that material classified as the most relevant was not necessarily the most reliable. They were more likely to assess the intended audience of the information first, to check that it was age appropriate. Information that was aimed at younger children was deemed lower quality;

"So they make it easier for them to understand, so they make it less detailed...so they can understand it on their level, like for their intelligence. And then they have it at an upper and more detailed [level]...for the adults" (Georgia, 15). 
In sum, whilst all participants valued easily accessible and relevant information, younger participants were primarily influenced by this when deciding whether information found online was of sufficient quality for their needs.

Website trustworthiness: Participants differed in the strategies used to evaluate trustworthiness of online information. The source of the information was particularly important for older participants. Websites of well-known medical institutions (e.g. local hospitals and the World Health Organization) were most trusted. Authorship by a healthcare professional was also seen as a key indicator of trustworthiness;

“...but what I like about this [example website] is that at the end, it says it's reviewed by this doctor...and if you click on it, then you can go 'oh, he works at...'” (Catherine, 23)

One way of judging the source was to inspect the website address for an indication of a medical or governmental institution. Those with a 'dot com' address were trusted less than those containing 'org' by one participant. She reasoned that “...usually governmental organizations have the correct information" and went on to say that "non-profit organizations....usually... they give trustworthy information" (Tabitha, 19).

The provision of references and sources of information enabled older participants to make a decision about its legitimacy. However, younger participants were more interested in the references or links as resources they could incorporate into their assignments, rather than as a sign of transparency. They did not appear to question the source of any information and when asked if they believed everything they read on Wikipedia, two participants said that they did. 
Participants discussed the fact that anyone could publish their views on a personal website, even without medical knowledge. When participants were asked to discuss the trustworthiness of a sample website, one participant said;

“It's kind of hard to say, because you don't really know whether [sic] this site originated from. Like, you don't know if it's based by doctors and researchers or if it's just a person who decided to make a website" (Georgia, 15)

However, despite identifying personal websites as potentially less trustworthy, participants connected strongly with first person narratives and found them a powerful source of information. Despite her previous concerns, when asked what type of information she wanted about health, Georgia said;

"Stories, like personal stories, or someone close to my age. So someone with my condition or something. Yeah, that's helpful.” (Georgia, 15).

Furthermore, participants of all ages talked about how their reaction to a website often guided them; e.g. "It just feels trustworthy" (Connor, 11). Tabitha (19) also talked about trusting information “...if it sounds correct”. Participants sometimes compared several websites to assess whether they concurred, going with the majority if in doubt.

When talking about security on the Internet, it became apparent that the younger participants had conflated the concepts of safety and reliability of information when talking about how their anti- 
virus software would ensure that they would always looked at reliable websites; "So if it's got a green check-mark, I trust it” (Joel, 14).

\section{Discussion}

\section{Summary of Findings}

Findings from Phase 1 demonstrated that there are relatively few websites about paediatric chronic conditions specifically aimed at children. Given the thousands of educational healthcare-related websites for adults, the small sample of equivalent websites for children in this study is quite remarkable, although, Stinson et al. (2009) also report that none of the 292 websites they identified about juvenile idiopathic arthritis were aimed specifically at children. Health care providers are clearly still not fully harnessing the benefits online information can potentially bring to young people with chronic conditions.

The reliability and quality of information on the websites demonstrated wide variation when using the DISCERN. Usual markers of reliable information, such as clear indications of the information source and its author, were often absent. Based on the average score for overall quality, most websites had "potentially important but not serious shortcomings”. According to previous classifications, the mean website summary score of 48.16 fell in the 'fair' (39-50) range (Lau et al, 2006), which has concerning implications for children and young people's use of online health information. It is of note that websites with a high reliability score (e.g. clear aims, author name, or date last updated), did not necessarily provide information which was inherently high quality, also reported by those examining adult online treatment information (Buhi et al., 2010; Bernstam et al., 2008). 
However, DISCERN does not allow for variables such as reading level, ease of navigation or multi-modal transmission, which are all likely to influence effective communication for children (Blowers \& Bryan, 2004). Thus, a website with extensive detail about treatment or lists of further reading, might receive a high DISCERN score despite being of little interest to the majority of younger users. These differing priorities need to be considered when designing both a website providing health information to children and an tool to evaluate such websites. Websites aimed at children need to incorporate sound pedagogical principles and positive learning strategies to provide cognitive support for a range of abilities and learning styles, which differs significantly from the majority of websites aimed at adults.

Though test-retest reliability was good (Gross Portnoy \& Watkins, 1999), the inter-rater reliability figures were lower than those reported with adult online information (Stinson et al., 2009; Ademiluyi et al., 2003). This indicates that DISCERN performed consistently when used by the same rater over time, but was inconsistent when used by multiple raters despite both using the DISCERN manual. Its internal consistency level (0.64) was also lower than the generally accepted level of 0.7 (Bland \& Altman, 1997). Therefore, we recommend that users be cautious if employing the DISCERN to evaluate information aimed at children.

Although some young adults may be able to use the DISCERN to rate the quality of websites (Stinson et al., 2009), it is likely that a simpler consumer guideline or tool is required to enable children of different ages and/or levels of development to use them optimally. However, we firstly need to understand what children consider to be high quality online information. Phase 2 of this study explored how children and youth assessed the quality and trustworthiness of online information. Our findings concur with limited previous work reporting that they often accept online information at 'face value', both generally and in relation to health (Hansen et al., 
2003), although older youth discussed identifying the source and author of the information as a way to judge trustworthiness. Despite this, youth also accepted information if it appeared vaguely credible. The younger participants did not evaluate the quality or trustworthiness of online information at all, instead giving priority to simple language, relevance, ease of access and provision of further resources.

\section{Strengths and limitations}

Very little research has explored the quality and reliability of online information about chronic conditions aimed at children rather than their parents. The evaluation of child-focused websites and exploration of how children and youth assess the trustworthiness and quality of online information detailed here contributes to the call for both the provision of high quality websites (Stinson et al., 2011) and empowering children and young people to carefully consider the health information they find online (Gray et al., 2005b; Moreno, Ralston, \& Grossman, 2009).

However, the study has a number of limitations. Only Google and Yahoo were used to search for relevant websites in Phase 1, potentially biasing the search. Nevertheless, these two search engines are unequivocally the most popular worldwide (StatCounter GlobalStats, 2010) and therefore represent the search engines health consumers would most likely use. This was also corroborated by the Phase 2 participants. Although the words 'youth' and 'young people' weren’t used explicitly as search terms, our additional strategy of using ‘snowball sampling’ (following links from one page to another) and directly contacting organizations ensured that we captured websites aimed at a wide range of different age-groups.

It could be argued that the low agreement values on the DISCERN between raters reflected inconsistent application of the tool. However, it is more likely to be due to the amount of interpretation required to complete the DISCERN for children's online information, as the tool 
does not accommodate information written for varying developmental stages. Therefore, each rater may have 'adjusted' the questions to apply to the website they were scoring, so that although they could be consistent within their own scoring, there was low uniformity between raters. Stinson et al report high inter-rater reliability rates between researchers when using the DISCERN with websites for adolescents with cancer (Stinson et al., 2011). However, the focus of their study was specifically to assess information on the diagnosis and treatment of cancer, which is much more in line with the original purpose of the DISCERN. This current study compared a wide range of chronic conditions with a huge diversity of management and treatment options, which made the consistent application of the tool between raters problematic. Whilst we believe our findings of variable website quality and reliability still hold true because of the high test-retest scores demonstrating consistency over time, there is a clear need for further work in this area.

The sample for Phase 2 was relatively small and primarily represented those with physical disabilities, despite purposive sampling to obtain children and young people with a range of conditions. A pragmatic decision was made to facilitate smaller groups so that children could participate fully and valuable data were not lost because facilitators were engaged elsewhere. We included children with a wide range of different ages, who revealed some marked differences in their views of Internet usage, which should be explored further in a wider sample to see how typical the participants in this study were. A large amount of cognitive and social development occurs during middle childhood through to adolescence (Bee \& Boyd, 2003) so exploring issues with the different age groups will help identify whether different tools are indeed required for children and young people due to developmental issues, such as topics of 
interest and appropriate level of detail. For example, older participants in this study reported assessing website quality by ascertaining the age of its intended audience.

Despite being exploratory, the study still strongly suggests that some children do not consider the trustworthiness of information found on the Internet at all, confirming previous findings that many children and young people routinely use the Internet to search for health information without necessarily having the skills to assess its trustworthiness or quality (Gray et al., 2005b; Kortum, Edwards, \& Richards-Kortum, 2008).

\section{Future Directions}

High quality websites are clearly needed for children and young people with chronic conditions and it is imperative that end users are involved with this process. The experience of using DISCERN with a wide range of health-related websites aimed at children indicates that an alternative tool is needed. Suggestions from older participants in Phase 2 indicate that a simple checklist with some key indicators of information trustworthiness (e.g. check the URL, identify if the website is associated with a hospital) may be a feasible strategy if researchers work closely with children and young people to generate and trial such concepts so that any tool is truly usercentred. Exploring how children use other sources of information, such as television, magazines and movies, may provide context around their appraisal of information and media literacy more generally. Further work is also needed around how children interpret potentially frightening or confusing material and their responses to it. It is likely that age and cognitive functioning, in addition to personal preference, are very important factors dictating children and young people's search for information, so developmentally appropriate items will have to be considered and extensively tested in any assessment tool. 


\section{Conclusion}

Many children and young people require greater skills for assessing the quality and trustworthiness of online health information. A brief assessment tool would have diverse applications, including use by healthcare professionals before referring clients to websites, or for independent use by children and their parents to evaluate information they find as they browse the web. Given that the Internet plays such a prominent role in today's society, children and youth should be able to identify high quality information about health and well-being, which may be particularly relevant to those living with a chronic condition.

\section{Competing Interests}

None.

\section{Acknowledgements}

We would like to thank the children and young people who took part in this study, as well as Sarah Keenan, Rebekah Keith and Mallory Ryan for their assistance. This project was funded by the Ward Family Foundation Summer Student Endowment Program, hosted by Bloorview Research Institute and a start-up grant from the Holland Bloorview Kids Rehabilitation Hospital Foundation. 


\section{Reference List}

Ademiluyi, G., Rees, C., \& Sheard, C. (2003). Evaluating the reliability and validity of three tools to assess the quality of health information on the Internet. Patient Education and Counseling, 50, 151-155.

Agency for Health Care Policy and Research. Criteria for assessing the quality of health information on the Internet. Available from: http://www.ahrq.gov/qual/hiirpt.htm. [Internet. Accessed May 1, 2009.]

Al-Bahrani, P. \& Plusa, S. (2004). The quality of patient-oriented internet information on colorectal cancer. Colorectal Disease, 6, 323-326.

Anselmo, M., Lash, K., Stieb, E., \& Haver, K. (2004). Cystic Fibrosis on the Internet: A Survey of Site Adherence to AMA Guidelines. Pediatrics, 114, 100-103.

Barlow, J. \& Ellard, D. (2004). Psycho-educational interventions for children with chronic disease, parents, and siblings: an overview of the research evidence base. Child: Care, Health and Development, 30, 637-645.

Barnes, M., Penrod, C., Neiger, B., Merrill, R., Thackeray, R., Eggett, D. et al. (2003). Measuring the relevance of evaluation criteria among health information seekers on the internet. Journal of Health Psychology, 8, 71-82.

Bee, H. \& Boyd, D. (2003). The developing child. (10th ed.) Boston, MA: Allyn \& Bacon. 
Bergman, J., Konijeti, R., \& Lerman, S. (2007). Myelomeningocele information on the Internet is accessible and of variable quality, and requires a high reading level. The Journal of Urology, 177, 1138-1142.

Bernstam, E., Walji, M., Sagaram, S., Sagaram, D., Johnson, C., \& Meric-Berstam, F. (2008). Commonly cited website quality criteria are not effective at identifying inaccurate online information about breast cancer. Cancer, 112, 1206-1213.

Blair, J. (2006). A computer and internet future: enabling inclusion? Learning Disability Practice, 9, 32-37.

Bland, J. \& Altman, D. (1997). Cronbach's alpha. BMJ, 314, 572.

Blowers, H. \& Bryan, R. (2004). Weaving a Library Web: A Guide to Developing children's Websites. Chicago: ALA.

Braun, V. \& Clarke, V. (2006). Using thematic analysis in psychology. Qualitative Research in Psychology, 3, 77-101.

Buhi, E., Daley, E., Obserne, A., Smith, S., Schneider, T., \& Fuhrmann, H. (2010). Quality and accuracy of sexual health information web sites visited by young people. Journal of Adolescent Health, 47, 206-208.

Charnock, D. \& Shepperd, S. (2004). Learning to DISCERN online: applying an appraisal tool to health websites in a workshop setting. Health Education Research, 19, 440-446.

Charnock, D. \& Shepperd, S. (2010). DISCERN Online. Radcliffe Online [On-line]. Available: http://www.discern.org.uk/index.php 
Charnock, D., Shepperd, S., Needham, G., \& Gann, R. (1999). DISCERN: an instrument for judging the quality of written consumer health information on treatment choices. Journal of Epidemiology and Community Health, 53, 105-111.

Dunn, D. \& Austin, J. (2004). Differential diagnosis and treatment of psychiatric disorders in children and adolescents with epilepsy. Epilepsy and Behavior, 5, S10-S17.

Erin Research Inc. (2005). Young Canadians in a wired world Media Awareness Network.

Franck, L., Noble, G., \& McEvoy, M. (2008). Enquiring minds want to know: Topics requested by users of a children's health information website. Patient Education and Counseling, 72, 168171.

Gray, N., Klein, J., Noyce, P., Sesselberg, T., \& Cantrill, J. (2005a). Health information-seeking behavior in adolescence: the place of the internet. Social Science and Medicine, 60, 1467-1478.

Gray, N., Klein, J., Noyce, P., Sesselberg, T., \& Cantrill, J. (2005b). The Internet: A window on adolescent health literacy. Journal of Adolescent Health, 37, 243.e1-243.e7.

Gross Portnoy, L. \& Watkins M.P. (1999). Foundations of Clinical Research: Applications to Practice. Lebanon: Prentice Hill.

Hanif, F., Read, J. C., Goodacre, J. A., Chaudhry, A., \& Gibbs, P. (2009). The role of quality tools in assessing reliability of the Internet for health information. Informatics for Health and Social Care, 34, 231-243.

Hansen, D., Derry, H., Resnick, P., \& Richardson, C. (2003). Adolescents searching for health information on the Internet: an observational study. Journal of Medical Internet Research, 5, e25. 
Hargrave, D., Hargrave, U., \& Bouffet, E. (2010). Quality of health information on the internet in pediatric neuro-oncology. Neuro-oncology, 8, 175-182.

Ilic, D., Risbridger, G., \& Green, S. (2004). Searching the internet for information on prostate cancer screening: an assessment of quality. Urology, 64, 112-116.

Kaicker, J., Borg Debono, V., Dang, W., Buckley, N., \& Thabane, L. (2010). Assessment of the quality and variability of health information on chronic pain websites using the DISCERN instrument. BMC Medicine, 8.

Kim P., Eng T.R., Deering M.J., Maxfield A. (1999). Published criteria for evaluating health related websites: review. British Medical Journal, 318, 647-9.

Kortum, P., Edwards, C., \& Richards-Kortum, R. (2008). The impact of inaccurate Internet health information in a secondary school learning environment. Journal of Medical Internet Research, 10, e17.

Kunst, H. (2002). Accuracy of information of apparently credible websites: survey of five common health topics. British Medical Journal, 324, 581-582.

Lathouwers, K., de Moor, J., \& Didden, R. (2009). Access to and use of internet by adolescents who have a physical disability: A comparative study. Research in Developmental Disabilities, 30, 702-711.

Lau, L., Hargrave, D.R., Bartels, U., Esquembre, C., Boufett, E. (2006). Childhood brain tumor information on the Internet in the Chinese language. Child's Nervous System, 22, 346-51. 
Leung, L. (2007). Stressful life events, motives for internet use, and social support among digital kids. Cyberpsychology, Behavior, and Social Networking, 10, 204-210.

Livingstone, S. \& Bober, M. (2005). UK Children go online: Final report of key project findings London: London School of Economics and Political Science.

McPherson, A., Glazebrook, C., \& Smyth, A. (2005). Educational interventions - computers for delivering education to children with respiratory illness and to their parents. Pediatric Respiratory Review, 6, 215-226.

McPherson, AC., Glazebrook, C., Forster, D., James, C., \& Smyth, A. (2006). A randomized, controlled trial of an interactive educational computer package for children with asthma. Pediatrics, 117, 1046-1054.

Montgomery, K. \& Chester, J. (2009). Interactive food and beverage marketing: targeting adolescents in the digital age. Journal of Adolescent Health, 45, S18-S29.

Moreno, M., Ralston, J., \& Grossman, D. (2009). Adolescent access to online health services: perils and promise. Journal of Adolescent Health, 44, 244-251.

Noriaki, A., Sachiko, O., Taisuke, O., Oishi, M., \& Tsuguya, F. (2005). INSULOT: A cellular phone-based edutainment learning tool for children with type 1 diabetes. Diabetes Care, 28, 760.

O'Connor, S. (2009). Coping with cancer: A web-based educational program for early and middle adolescents. Journal of Pediatric Oncology Nursing, 26, 230-241.

Pantell, R. \& Lewis, C. (1993). Talking with children: how to improve the process and outcomes of medical care. Medical Encounter, 10, 3-7. 
Rushforth, H. (1999). Practitioner review: communicating with hospitalised children: review and application of research pertaining to children's understanding of illness. Journal of Child Psychology and Psychiatry, 40, 683-691.

Sawyer, S., Drew, S., Yeo, M., \& Britto, M. (2007). Adolescents with a chronic condition: challenges living, challenges treating. Lancet, 369, 1481-1489.

Silberg, W., Lundberg, G., \& Musacchio RA (1997). Assessing, controlling, and assuring the quality of medical information on the internet. Journal of the American Medical Association, 277, $1244-1245$.

Standen, P. (1998). Playing for real. Mental Health Care, 1, 412-415.

StatCounter GlobalStats (2010). Top 5 Search Engines from Jul 09 to Aug 10. StatCounter GlobalStats [On-line]. Available: http://gs.statcounter.com/\#search_engine-ww-monthly$\underline{200907-201008}$

Stewart, M., Barnfather, A., Magill-Evans, J., Ray, L., \& Letourneau, N. (2011). Brief report: An online support intervention: Perceptions of adolescents with physical disabilities. Journal of Adolescence, 34, 795-800.

Stieb, E., Wang, J., \& Haver, K. (2002). Do food allergy websites satisfy AMA guidelines for health information on the internet? Journal of Allergy and Clinical Immunology, 109, S309.

Stinson, J., Tucker, L., Huber, A., Harris, H., Lin, C., Cohen, L. et al. (2009). Surfing for Juvenile Idiopathic Arthritis: Perspectives on quality and content of information on the internet. British Journal of Rheumatology, 36, 1755-1762. 
Stinson, J. N., White, M., Breakey, V., Chong, A. L., Mak, I., Low, K. K. et al. (2011).

Perspectives on quality and content of information on the internet for adolescents with cancer. Pediatric Blood \& Cancer, 57, 97-104.

Suris, J.-C., Akre, C., Berchtold, A., Belanger, R., \& Michaud, P.-A. (2010). Chronically connected? Internet use among adolescents with chronic conditions. Journal of Adolescent Health, 46, 200-202.

Swift, J. \& Tischler, V. (2010). Qualitative research in nutrition and dietetics: getting started. Journal of Human Nutrition and Dietetics, 23, 559-566.

The Pew Foundation (2010). Change in Internet access by age group, 2000-2010.

Tieffenberg, J., Wood, E., Alonso, A., Tossutti, M., \& Vicente, M. (2000). A randomised field trial of ACINDES: a child-centred training model for children with chronic illness (asthma and epilepsy). Journal of Urban Health, 77, 280-297.

Torpy, J., Lynm, C., \& Glass, R. (2007). Chronic disease of children. Journal of the American Medical Association, 207, 2836.

Veldtman, G., Matley, S., Kendall, L., Quirk, J., Gibbs JL, Parsons, J. et al. (2000). Illness understanding in children and adolescents with heart disease. Heart, 84, 395-397.

Wong, S. \& Tam, S.-F. (2010). Effectiveness of a multimedia programme and therapistinstructed training for children with autism. International Journal of Rehabilitation Research, 24, 269-278.

Wood, D (1998). How children think and learn. Oxford: Blackwell. 
Yeo, M. \& Sawyer, S. (2005). Chronic illness and disability. British Medical Journal, 330, 721723. 


\section{Table 1- DISCERN Questions}

\begin{tabular}{|c|c|}
\hline \multicolumn{2}{|l|}{ Section 1} \\
\hline Question 1 & Are the aims clear? \\
\hline Question 2 & Does it achieve its aims? \\
\hline Question 3 & Is it relevant? \\
\hline Question 4 & $\begin{array}{l}\text { Is it clear what sources of information were used to } \\
\text { compile the publication (other than the author or } \\
\text { producer)? }\end{array}$ \\
\hline Question 5 & $\begin{array}{l}\text { Is it clear when the information used or reported } \\
\text { in the publication was produced? }\end{array}$ \\
\hline Question 6 & Is it balanced and unbiased? \\
\hline Question 7 & $\begin{array}{l}\text { Does it provide details of additional sources of } \\
\text { support and information? }\end{array}$ \\
\hline Question 8 & Does it refer to areas of uncertainty? \\
\hline \multicolumn{2}{|l|}{$\underline{\text { Section } 2}$} \\
\hline Question 9 & Does it describe how each treatment works? \\
\hline Question 10 & Does it describe the benefits of each treatment? \\
\hline Question 11 & Does it describe the risks of each treatment? \\
\hline Question 12 & $\begin{array}{l}\text { Does it describe what would happen if no treatment } \\
\text { is used? }\end{array}$ \\
\hline Question 13 & $\begin{array}{l}\text { Does it describe how the treatment choices affect } \\
\text { overall quality of life? }\end{array}$ \\
\hline Question 14 & Is it clear that there may be more than one possible treatment choice? \\
\hline Question 15 & Does it provide support for shared decision-making? \\
\hline
\end{tabular}




\section{$\underline{\text { Section } 3}$}

Question 16

Based on the answers to all of the above questions,

rate the overall quality of the publication as a source

of information about treatment choices

Note: Only Sections 1-2 were summed to assign the overall score. Section 3 (Question 16) was excluded from data analysis. 
Table 2: Index of Website Topics (numbers indicate frequency within sample)

\begin{tabular}{|c|c|c|c|}
\hline 1 & Acquired Brain Injury & 2 & HIVIAIDS \\
\hline 1 & Anemia [not SCA - below] & 2 & IBD (Chron's disease or Ulcerative Colitis) \\
\hline 1 & Anxiety Disorders & 2 & Muscular Dystrophy \\
\hline 1 & Bacterial Meningitis & 2 & Peanut Allergy \\
\hline 1 & Bipolar Disorder & 3 & ADHD \\
\hline 1 & Celiac Disease & 3 & Cystic Fibrosis \\
\hline 1 & Duchenne Muscular Dystrophy & 3 & Irritable Bowel Syndrome \\
\hline 1 & Ehlers-Danlos syndrome & 3 & Obesity \\
\hline 1 & Juvenile Rheumatoid Arthritis & 3 & Tourette Syndrome \\
\hline 1 & Kidney Disease & 4 & Cerebral Palsy \\
\hline 1 & Obsessive-Compulsive Disorder & 4 & Scoliosis \\
\hline 1 & Restrictive cardiomyopathy & 4 & Spina Bifida/Spina Bifida \& Hydrocephalus \\
\hline 1 & Sickle Cell Anemia & 4 & Autism \\
\hline 2 & Allergies & 6 & Dyslexia \\
\hline 2 & Asperger's Syndrome & 9 & Asthma \\
\hline 2 & Cancer & 12 & Diabetes (Type I and/or II) \\
\hline 2 & Down Syndrome & 13 & Epilepsy \\
\hline
\end{tabular}


Table 3- Participants’ demographics (*all names are pseudonyms).

\begin{tabular}{|c|c|c|c|c|}
\hline Participant* & $\begin{array}{c}\text { Workshop } \\
\#\end{array}$ & Age & Condition & $\begin{array}{c}\text { Hours of } \\
\text { internet use } \\
\text { per week }\end{array}$ \\
\hline Kristen & 1 & 18 & Cerebral palsy & $7+$ \\
\hline Georgia & 1 & 15 & Cerebral palsy & $1-2$ \\
\hline Catherine & 1 & 23 & $\begin{array}{l}\text { Cerebral } \\
\text { palsy/scoliosis/spina } \\
\text { bifida }\end{array}$ & $6-7$ \\
\hline Tabitha & 2 & 19 & Acquired brain injury & $7+$ \\
\hline Connor & 2 & 11 & Cerebral palsy & $5-6$ \\
\hline Joel & 2 & 14 & $\begin{array}{l}\text { Attention Deficit } \\
\text { Disorder (sibling) }\end{array}$ & $5-6$ \\
\hline
\end{tabular}

